\title{
Removal of Power Line Interference in ECG Signal by Adaptive LMS, NLMS and RLS Algorithms
}

\author{
P. Pavani ${ }^{1}$, N.V.R. Adithya ${ }^{2}$, K. Hari Krishna ${ }^{3}$, M.V. Siva Kumar ${ }^{4}$ \\ Assistant Professor, ECE, GVP College of Engineering, Visakhapatnam, India ${ }^{1}$ \\ B. Tech., ECE, GVP College of Engineering, Visakhapatnam, India ${ }^{2,3,4}$
}

\begin{abstract}
Filtering of power line interference is very meaningful in the measurement of biomedical events recording, particularly in the case of recording signals as weak as the ECG signal. ECG is a biomedical signal which gives electrical activity of heart. This ECG signal is corrupted by various noises like power line interference, baseline wandering, channel noise, contact noise, muscle artifacts etc. Frequency range of ECG signal is nearly same as the frequency of power line interference. ECG signal has frequency range from $0.5 \mathrm{~Hz}$ to $80 \mathrm{~Hz}$ and power line interference introduces 50 to $60 \mathrm{~Hz}$ frequency component in that signal which is the major cause of corruption of ECG. From various artifacts contaminate electrocardiogram (ECG) recording, the most common are power line interference. Power line interference is not easily recognizable since the interfering voltage in the ECG may have frequency $50 \mathrm{~Hz}$. The most common cause of $50 \mathrm{~Hz}$ interference is the disconnected electrode resulting in a very strong disturbing signal, and therefore needs quick action. Electromagnetic interference from the power lines also results in poor quality tracings. Electrical equipment's such as air conditioner, elevators and X-ray units draw heavy power line current, which induce $50 \mathrm{~Hz}$ signals in the input circuits of the ECG machine. This project focuses on a technique for de noising of such non stationary ECG signal using adaptive filter algorithms like LMS, NLMS, RLS and compares their performance characteristics using MATLAB.
\end{abstract}

Keywords: ECG, LMS, NLMS, RLS, MATLAB, Power Line Interference.

\section{INTRODUCTION}

The main function of the heart is to pump blood throughout the body to deliver the oxygen and nutrient demands of the body's tissues as well as to remove carbon dioxide. There are various biomedical signals present in the human body, by examining these biomedical signal one can check the health condition whether that person is clinically fit or not. Electrocardiogram is one of them. ECG signal is electric representation of the activity of human's heart. Various Cardiac diseases can be recognized with the help of ECG Signal.

A typical ECG signal is shown in figure 1 which is composed of individual P, Q, QRS Complex, S, T, U waves. Each individual component has its own amplitude and interval range which helps in determining the activity of heart. For example, $\mathrm{P}$ wave which is generated due to electrical activity of atria and $\mathrm{T}$ wave represent re-polarization of ventricles. Similarly each wave has its own function in determining the electrical activity of heart by ECG signal.

While recording process of ECG signal, several types of noises may encounter in it. The major type of noise, this project deals with is Power Line Interference (PLI).

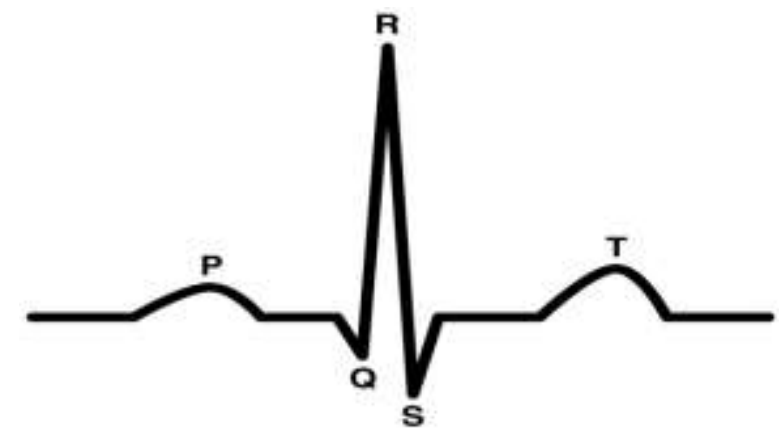

Fig. 1. ECG Signal 
The objective of this report is to separate the valid signal component from the undesired noises so that the accurate interpretation of ECG signals could be done.

Recently, Adaptive filtering has become one of the effective and popular methods for the processing and analysis of the ECG signal because of its non-stationary nature. And in this study, Adaptive LMS, NLMS, and RLS algorithms are used to filter power line interference from the ECG signal and compare their performance based on SNR value [1]-[4].

\section{MAJOR NOISES EFFECTING ECG SIGNAL}

A. Power Line Interference:

The Power Line interference of $50-60 \mathrm{~Hz}$ is the source of interference and it corrupts the recordings of ECG, which plays a major role in the diagnosis of the patient. The causes for Power Line interference are-

1. Electromagnetic interference by power line

2. Stray effect of the alternating current due to loops in the cables.

3. Improper grounding of ECG machine or the patient

Power Line interference signal generated using MATLAB is shown in figure 4.

B. Electromyography (EMG) Noise:

EMG noise is caused by the contraction of other muscles besides the heart. When other muscles in the vicinity of the electrodes contract, they generate depolarization and repolarization waves that can also be picked up by the ECG. The extent of the crosstalk depends on the amount of muscular contraction.

\section{Instrumentation Noise:}

The electrical equipment used in ECG measurements also contributes noise. The major sources of this form of noise are the electrode probes, cables, signal processor/amplifier.

\section{Channel Noise:}

Idle-Channel noise is the noise present in the communication channel when no signal is applied. Poor channel conditions can also introduce noise to the ECG, when ECG is transmitted. It is mainly like white Gaussian noise which contains all frequency components

\section{III.ADAPTIVE FILTER}

An adaptive filter is a filter that self-adjusts its transfer function according to an optimization algorithm driven by an error signal. Because of the complexity of the optimization algorithms, most adaptive filters are digital filters. The adaptive filter reduces the mean squared error between primary input (ECG signal) and the reference input (noise with ECG signal). The power line interference $(50 \mathrm{~Hz})$ from ECG signal can be removed by adaptive filtering. Fixed filter designers assume that the signal characteristics of the statistical computing environment fully known, it must be based on the prior knowledge of the signal and noise. However, in most cases it is very difficult to meet the conditions; most of the practical issues must be resolved using adaptive filter.

The word adaptive means to adjust with other environment (system) by having the same response as the system itself to some phenomenon which is taking place in its surroundings or the system which tries to adjust its parameter, depending upon the other system's behavior and its surrounding. The systems which carries out its functionality after undergoes the process of adaptation is called filter [5].

General Adaptive filter which uses the digital filter is shown in figure 2. $\mathrm{x}(\mathrm{n})$ is the input given to adaptive filter and $y(n)$ is the output of filter. Adaptive algorithm adjusts the filter coefficient included in the vector $w(n)$, in order to let the error signal e (n) to be the s malle st. Error signal is the difference of useful signal d(n) and the filter output $y(n)$.

Therefore, adaptive filter automatically carry on a design based on the characteristic of the input signal $\mathrm{x}(\mathrm{n})$ and the useful signal d (n). Using this method, adaptive filter can be adapted to the environment set by these signals. When the environment changes, filter through a new set of factors, adjusts for new features. The most important property of adaptive filter is that it can work effective in unknown environment, and to track the input signal of time-varying characteristics. Adaptive filter is classified into two parts, adaptive algorithm and digital filter. Function of adaptive algorithm is making proper filter coefficient. General digital filters use fixed coefficients, but adaptive filter change filter coefficients in consideration of input signal, environment, and output signal characteristics. 


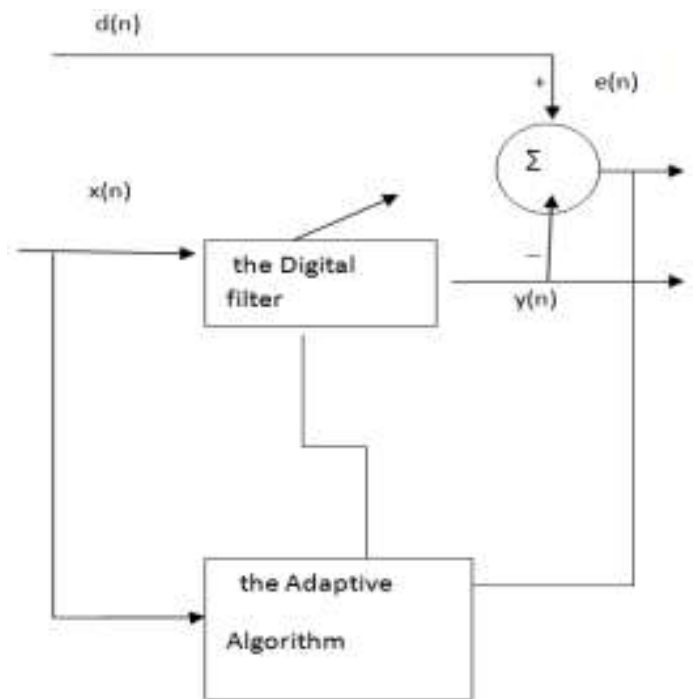

Fig. 2. Adaptive Filter

\section{A. LMS Algorithm}

Least mean squares (LMS) algorithms are class of adaptive filter used to mimic a desired filter by finding the filter coefficients that relate to producing the least mean squares of the error signal (difference between the desired and the actual signal). The basic idea behind LMS filter is to approach the optimum filter weights, by updating the filter weights in a manner to converge to the optimum filter weight. The algorithm starts by assuming a small weights (zero in most cases), and at each step, by finding the gradient of the mean square error, the weights are updated [1]. That is, if the MSE-gradient is positive, it implies, the error would keep increasing positively, if the same weight is used for further iterations, which means we need to reduce the weights. In the same way, if the gradient is negative, we need to increase the weights. So, the basic weight update equation is:

$$
\mathrm{w}(\mathrm{n}+1)=\mathrm{w}(\mathrm{n})+\mu^{*} \mathrm{x}(\mathrm{k}) * \mathrm{e}(\mathrm{n})
$$

Where e (n) is the error signal

$$
e(n)=y(n)-d(n)
$$

Where $d(n)$ is output of the filter given by

$$
\mathrm{d}(\mathrm{n})=\mathrm{w}(\mathrm{n})^{\mathrm{T}} * \mathrm{x}(\mathrm{n})
$$

\section{Importance of $\mu$ and $n$ :}

Step size $(\mu)$ is critical for the update and must be chosen accurately to ensure the filter converges. Updating the filter coefficients is important because this is the part of the code that governs how well the filter will converge to the desired response. Another element that has a key role in this convergence is the number of filter coefficients n, intuitively the number of coefficients must at least equal the length of the impulse response of the unknown system.

If $\mu$ is chosen to be very small then the algorithm converges very slowly. A large value of $\mu$ may lead to a faster convergence but may be less stable around the minimum value.

\section{B. NLMS Algorithm}

The main drawback of the "pure" LMS algorithm is that it is sensitive to the scaling of its input. This makes it very hard to choose a learning rate $\mu$ that guarantees stability of the algorithm. The Normalized least mean squares (NLMS) filter's a variant of the LMS algorithm that solves this problem by normalizing with the power of the input.

Normalized Least Mean Square (NLMS) is actually derived from Least Mean Square (LMS) algorithm. The need to derive this NLMS algorithm is that the input signal power changes in time and due to this change the step-size between two adjacent coefficients of the filter will also change and also affect the convergence rate. Due to small signals this convergence rate will slow down and due to loud signals this convergence rate will increase and give an error. So to overcome this problem, try to adjust the step-size parameter with respect to the input signal power. Therefore while designing the LMS adaptive filter, one difficulty we meet is the selection of the step-size parameter $\mu$. Therefore the step-size parameter is said to be normalized.

When the convergence factor $\mu$ is large, the algorithm experiences a gradient noise amplification problem. In order to solve this difficulty, the NLMS (Normalized Least Mean Square) algorithm is used. The correction applied to the 
weight vector $\mathrm{w}(\mathrm{n})$ at iteration $\mathrm{n}+1$ is "normalized" with respect to the squared Euclidian norm of the input vector $\mathrm{x}(\mathrm{n})$ at iteration $n$ [4]. Basic weight update equation in NLMS is

$$
\begin{gathered}
\mathrm{w}(\mathrm{n}+1)=\mathrm{w}(\mathrm{n})+\left(\mu^{*} /\left(\mathrm{x}^{\mathrm{T}}(\mathrm{n}) * \mathrm{x}(\mathrm{n})\right)^{*} \mathrm{e}(\mathrm{n}) * \mathrm{x}(\mathrm{n})\right. \\
\text { Normalized step size } \mu^{\prime}=\mu /\left(\mathrm{x}^{\mathrm{T}}(\mathrm{n}) * \mathrm{x}(\mathrm{n})\right)
\end{gathered}
$$

Where e (n) is error signal

$$
e(n)=y(n)-w(n)^{T} * x(n)
$$

C. RLS Algorithm

Recursive least squares (RLS) adaptive filter is an algorithm which recursively finds the filter coefficients that minimize a weighted linear least squares cost function relating to the input signals. The RLS algorithms are known for their excellent performance when working in time varying environments but at the cost of an increased computational complexity and some stability problems.

The idea behind RLS filters is to minimize a cost function C by appropriately selecting the filter coefficients $\mathrm{w}$ ( $\mathrm{n}$ ), updating the filter as new data arrives [6].

The equation for the cost function $\mathrm{C}$

$$
\mathrm{C}\left(\mathrm{w}_{\mathrm{n}}\right)=\sum_{i=0}^{n} e^{2}(\mathrm{i}) \lambda^{n-i}
$$

Inorder to minimise the cost function $\mathrm{C}$, We must take partial derivatives of $\mathrm{C}$ with respect to weights and equal it to zero. By solving that partial differentiation equation, updated weight equation of the filter using RLS algorithm is as below.

$$
\begin{gathered}
\mathrm{w}(\mathrm{n}+1)=\mathrm{w}(\mathrm{n})+\mathrm{k}(\mathrm{n}) * \mathrm{e}(\mathrm{n}) \\
\mathrm{k}(\mathrm{n})=\lambda^{-1} * \mathrm{P}(\mathrm{n}) * \mathrm{x}(\mathrm{n}) /\left(1+\lambda^{-1 *} \mathrm{x}(\mathrm{n})^{\mathrm{T}} * \mathrm{P}(\mathrm{n}) * \mathrm{x}(\mathrm{n})\right) \\
\mathrm{P}(\mathrm{n})=\lambda^{-1} * \mathrm{P}(\mathrm{n})-\lambda^{-1} * \mathrm{P}(\mathrm{n}) * \mathrm{x}(\mathrm{n}) /\left(1+\lambda^{-1} * \mathrm{x}(\mathrm{n}){ }^{\mathrm{T}} * \mathrm{P}(\mathrm{n}) * \mathrm{x}(\mathrm{n})\right)
\end{gathered}
$$

Where $\lambda$ is called forgetting factor. The smaller $\lambda$ is, the smaller is the contribution of previous samples to the covariance matrix $R_{x}(n)$. Where $R_{x}(n)$ is sample covariance matrix for $x(n)$.

The filter output is calculated using the filter tap weights of iteration and the current input vector as in equation below

$$
\mathrm{y}(\mathrm{n})=\mathrm{w}^{\mathrm{T}}(\mathrm{n}-1) * \mathrm{x}(\mathrm{n})
$$

Error signal e(n)

$$
e(n)=d(n)-y(n)
$$

\section{IV.SIMULATION RESULTS}

The original ECG signal is generated by using MATLAB as shown in the figure. The $50 \mathrm{~Hz}$ power line interference is also generated with sampling frequency of $2000 \mathrm{~Hz}$ is shown in figure. The power line interference is then added to the original ECG signal to get the mixed signal as shown in figure. Finally, the power line interference is removed using different adaptive filters based on different algorithms. The simulation results for filters using LMS, NLMS and RLS algorithms are obtained using MATLAB software are shown here

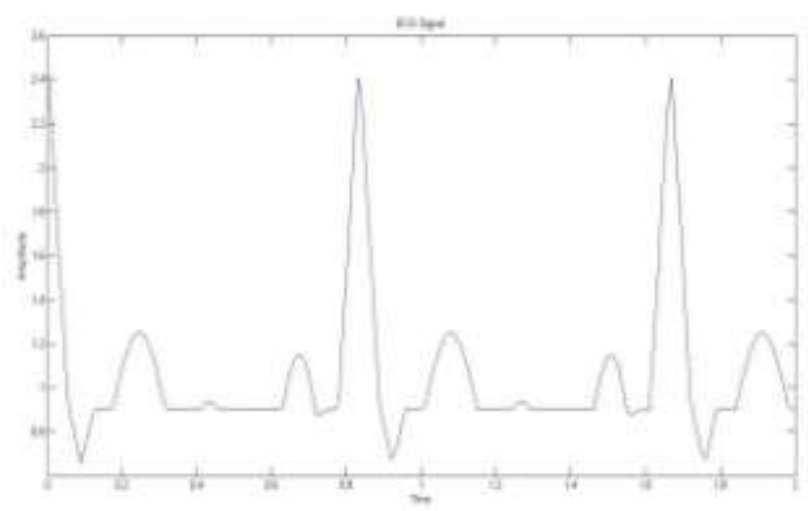

Fig. 3. Pure ECG Signal 


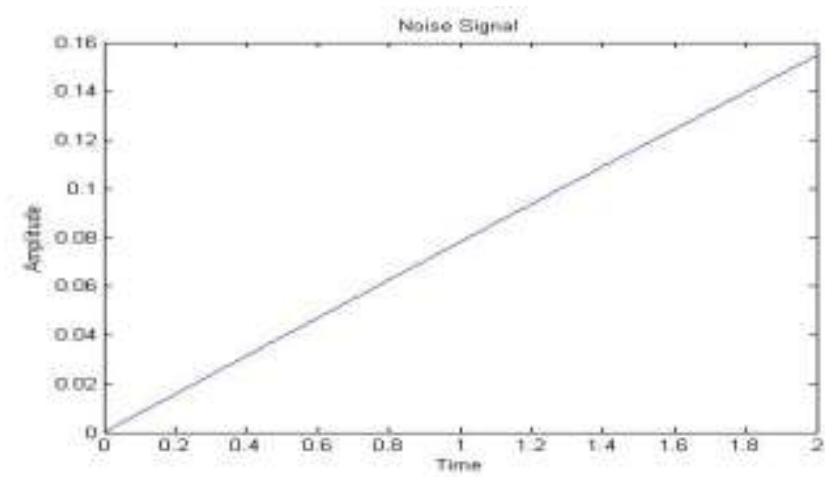

Fig. 4. Power Line interference signal

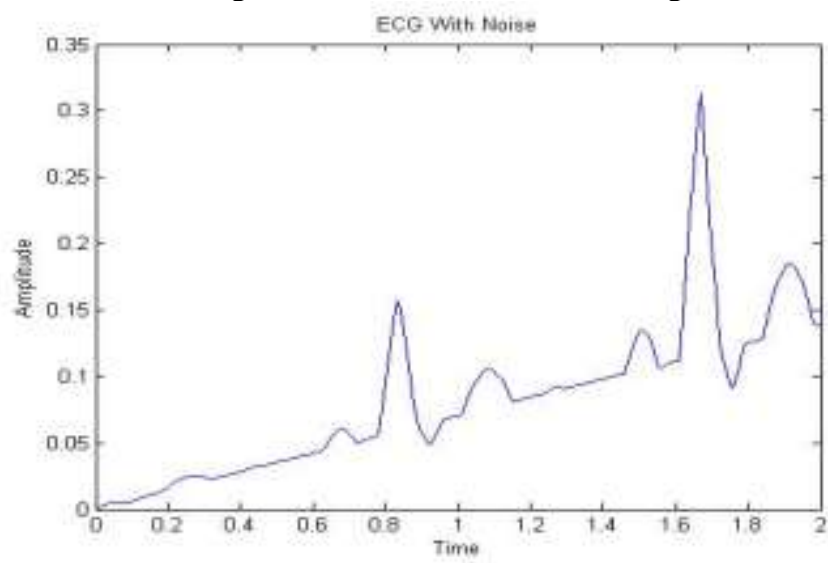

Fig. 5. ECG Signal with PLI

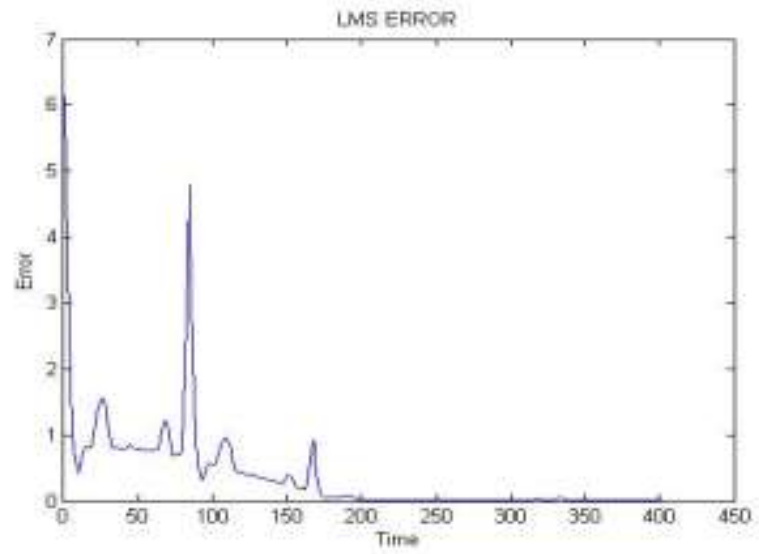

Fig. 6. Error Signal of filter using LMS Algorithm

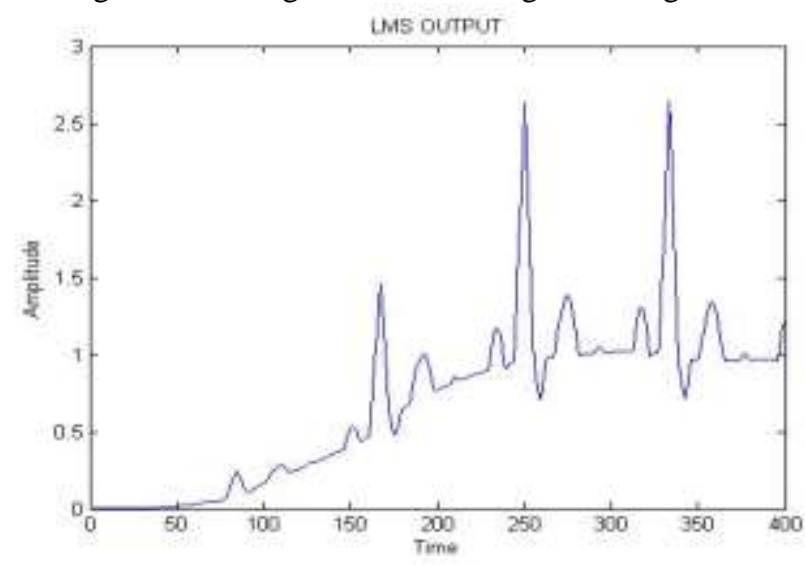

Fig. 7. Output of the filter using LMS Algorithm 


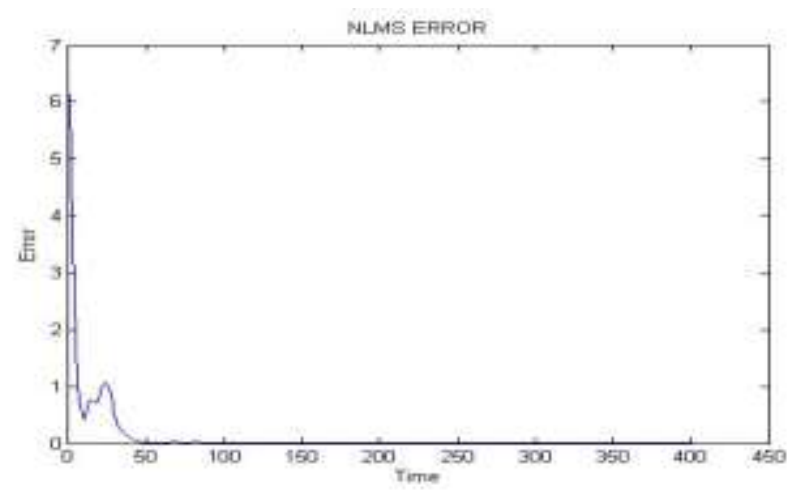

Fig. 8. Error Signal of filter using NLMS Algorithm

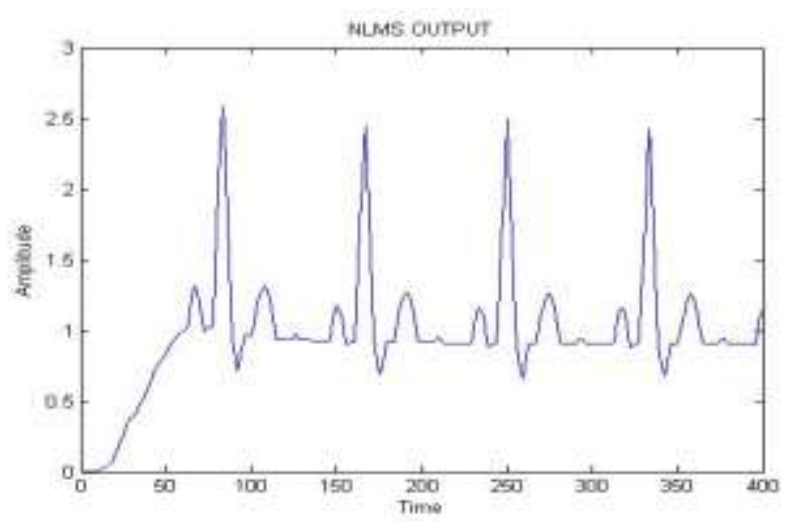

Fig. 9. Output of the filter using NLMS Algorithm

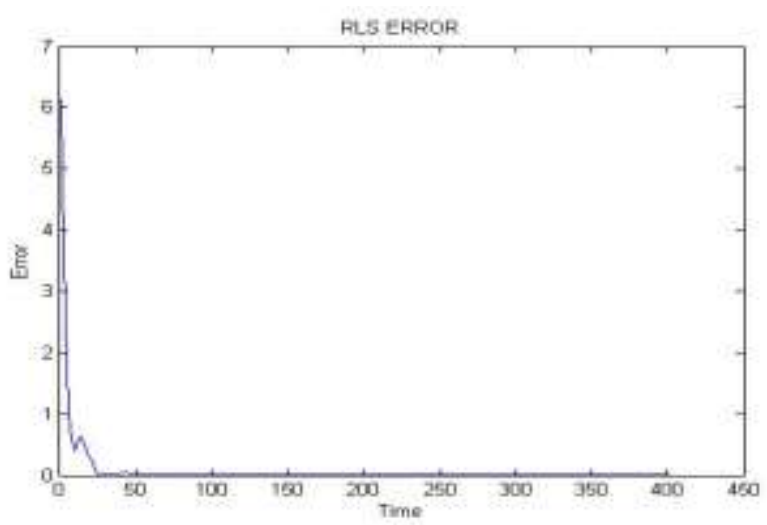

Fig. 10. Error signal of the filter using RLS Algorithm

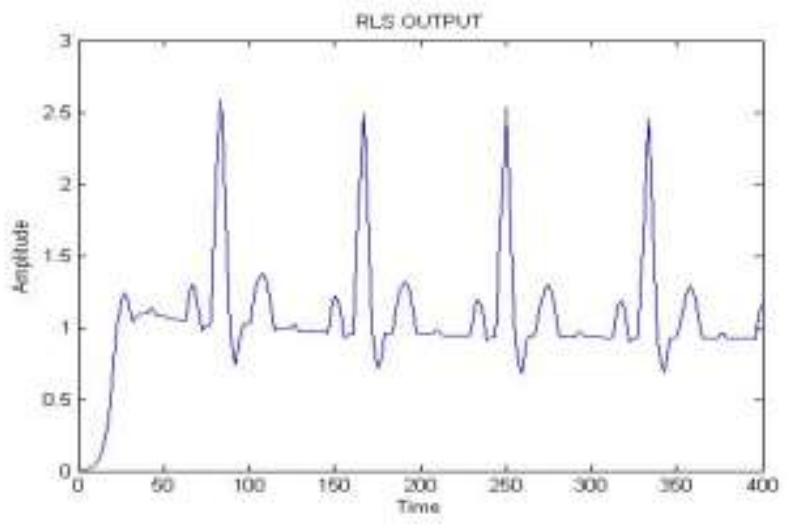

Fig. 11. Output of the filter using RLS Algorithm 


\section{COMPARISON OF SNR VALUES FOR LMS, NLMS, RLS ALGORITHMS}

Table 1. SNR Values before and after filtering

\begin{tabular}{|l|l|l|}
\hline Algorithm used in filter & \multicolumn{1}{|c|}{ SNR value before filtering } & \multicolumn{1}{c|}{ SNR value after filtering } \\
\hline LMS & 1.1566 & 1.9719 \\
\hline NLMS & 1.1566 & 10.7697 \\
\hline RLS & 1.1566 & 16.4313 \\
\hline
\end{tabular}

\section{VI.CONCLUSION}

The main objective of this project was to implement an adaptive noise canceller for de-noising an ECG signal and test the performance of the system for various algorithms. RLS algorithm proved to have the highest convergence speed and higher SNR improvement but at the cost of large computational complexity and memory requirements. The NLMS algorithm changes the step size according to the energy of the input signals hence it is suitable for both stationary as well as non-stationary environment and its performance lies between LMS and RLS. The main drawback of the "pure" LMS algorithm is that it is sensitive to the scaling of its input. This makes it very hard to choose a step size $\mu$ that guarantees stability of the algorithm.

\section{REFERENCES}

[1] H.N Bharath, K.M.M Prabhu," A New LMS based Adaptive Interference Canceller for ECG Power Line Removal", International Conference on Biomedical Engineering (ICoBE), February 2012

[2] N.M.Verulkar, P. H. Zope, S. R. Suralkar, "Filtering Techniques for Reduction of Power Line Interference in Electrocardiogram Signals.", International Journal of Engineering Research \& Technology (IJERT) Vol. 1 Issue 9, November- 2012 ISSN: $2278-0181$.

[3] A.Bhavani Sankar, D.Kumar \& K.Seethalakshmi," Performance Study of Various Adaptive Filter Algorithms for Noise Cancellation in Respiratory Signals", Signal Processing: An International Journal (SPIJ), Volume (4): Issue (5).

[4] J. M. Griz, 1. Raez, S. C. Alvarez, C. G. Puntonet, E.W. Lang and Deniz. E, "A Novel LMS Algorithm Applied to Adaptive Noise Cancellation, IEEE Signal Processing Letters" vol. 16, pp 34-37, Jan, 2009.

[5] Dr. Shaila D. Apte, Adaptive FIR \& IIR Filter design, Advanced Digital Signal Processing, Wiley, 2016:156-160,218-222.

[6] Theodore S. Rappaport,, Algorithms for Adaptive Filters, Wireless Communications, Prentice Hall Communications Engineering and Emerging Technologies Series:372-390. 